\title{
Alice Springs, die Oase im Herzen Australiens
}

\author{
Werner Nigg
}

\section{Die erste Telegraphenstation}

Im Jahre 1872 wurde die Úberland-Telegraphenlinie von (Adelaide-) Port Augusta nach Darwin in Betrieb genommen und gleichzeitig an die Unterwasserkabellinie nach Batavia-Singapore angeschlossen. Damit war das abgelegene Australien der übrigen Welt wieder um einen Schritt näher gerückt. Im Zusammenhang mit dem Bau und Betrieb dieser mehr als $3000 \mathrm{~km}$ langen Transkontinentalleitung entstand auf halbem Weg, im Zentrum Australiens - rund $30 \mathrm{~km}$ südlich des Wendekreises des Steinbocks - eine Telegraphenstation. Ihr Standort wurde an einer Stelle gewählt, wo man in einem trockenen, sandigen Flußbett einige Wasserlöcher mit herrlich klarem, frischen Grundwasser fand - ein kostbares Gut in dieser Region. Der Fluß, der nur selten Wasser führt, erhielt nach dem damaligen Generalpostmeister und Betriebsdirektor der Telegraphenlinie, Sir Charles Todd, den Namen Todd River; und die Wasserquellen nannte man nach dessen Gattin, Lady Alice Todd, Alice Springs. Niemand ahnte damals, daß dieser Name einige Dezennien später von einer der eigenartigsten Siedlungen des Kontinents übernommen werden sollte.

Die kleine Siedlung der Telegraphenstation bestand aus einem halben Dutzend Steinhäusern, in denen das Telegraphen- und Postbüro, die Polizeiwache, Wohn- und Vorräteräume, Stallungen und Wagenremisen untergebracht waren. Jahrelang führten die wenigen Bewohner der Telegraphenstation ein einsames Dasein. Einige hundert scheue Aborigines und wenige Weiße, die im weiten Umkreis auf zerstreuten Schaffarmen lebten, waren die einzigen Menschen in dieser immensen, unwirtlichen Gegend, deren Bevölkerungsdichte auch heute noch zu den geringsten der ganzen Erde gehört (1 Einwohner auf 20 Quadratkilometer). Trotz der Großartigkeit der Naturlandschaft mit ihren weiten Ebenen, nackten, roten Bergrücken, den spärlichen niederen Busch- und Steppensträuchern und einigen Galeriewäldern mit prachtvollen Eukalypten, sehnten sich die Telegraphenbeamten und deren Angehörige schon nach wenigen Jahren nach den lebhaften, mehr Abwechslung bietenden Küstenstädten Adelaide, Melbourne, Sydney oder Brisbane.

\section{Das Klima}

Das Klima dieser zentralaustralischen Region ist heiß und trocken. Während vieler Tage des Jahres wölbt sich ein wolkenloser Himmel über die weite Landschaft. Um die Weihnachtszeit brennt über Mittag die erbarmungslose Sonne senkrecht herunter, und die wenigen Menschen bleiben in den kühlen Häusern, dieweil die Tiere versuchen, in den spärlichen kleinen Schattenflecken der Bäume und Sträucher Schutz vor den glutheißen Strahlen zu finden. Im Sommer steigen die Tagestemperaturen nicht selten bis auf $46^{\circ} \mathrm{C}$. Die Hitze ist aber wegen der Trockenheit der Atmosphäre verhältnismäßig leicht zu ertragen. In den klaren Nächten kühlt sich die Luft angenehm ab. Die mittlere Januar-(Sommer)temperatur beträgt $28^{\circ} \mathrm{C}$, die durchschnittliche Juli-(Winter)temperatur $12^{\circ} \mathrm{C}$. Selten fegen heftige Sandstürme über das Land, verdunkeln den Himmel und hüllen alles in Grau. Dann dringen die feinen Staubteilchen durch alle Ritzen und Kleider. Hie und da folgen diesen Stürmen wolkenbruchartige Gewitterregen, und durch die sonst trockenen Bach- und Flußbette wälzen sich gewaltige, schlammige Wasserwogen. Doch nach kurzer Zeit, nicht selten schon nach wenigen Minuten, bricht die Sonne durch die Wolken, im Nu ist der Boden wieder trocken, und die Bäche sind nur noch harmlose Rinnsale. Solche Regenfälle sind über das ganze Jahr verteilt, kommen indessen im Südsommer häıfiger vor als im Südwinter. Sie sind jedoch so selten, daß die mittleren Regenmengen sehr gering sind, diese betragen im Januar 40, im Juli 10 und im Jahr $286 \mathrm{~mm}$.

\section{Township of Stuart}

Schon 1888 begann man $4 \mathrm{~km}$ südlich der Telegraphenstation in einer von einigen Hügeln durchsetzten Ebene mit der Planung und dem Bau der "Township of Stuart», benannt nach John McDouall Stuart, der als einer der ersten Zentralaustralien durchquert hat. Hier fand man in etwa $30 \mathrm{~m}$ Tiefe reichlich Grundwasser, das mittels artesischer Brunnen leicht erschlossen werden konnte. Es gibt auch heute kaum ein schmackhafteres Trinkwasser in Australien, als jenes dieses artesischen Beckens. Südlich der Ebene, die etwa $650 \mathrm{~m}$ ü.M. liegt, liegen 
in Ost-West-Richtung die MacDonnell Ranges: diese bis $1000 \mathrm{~m}$ hohen, fast gänzlich vegetationslosen Höhenzüge bestehen aus rötlichen vorkambrischen, meist kristallinen Gesteinen und bieten besonders im Südwinter einigermaßen Schutz vor den Südostwinden. Durch diesen Bergzug hat der Todd River den Heavitree Gap, eine enge Klus, eingeschnitten, in der heute neben dem Flußbett die Straße und Bahnlinie gerade noch knapp Platz finden. Auch im Norden bilden einige Ost-West verlaufende Höhenzüge den Abschluß der Ebene.

Stuart wurde wichtiger Etappenort der Nord-SüdRoute mit Hotels, Bars, Ladengeschäften, und bald war es Ausgangspunkt für die Erforschung Zentralaustraliens. Immer häufiger kamen Anthropologen und Völkerkundler, um die Aborigines zu studieren; Biologen, um die eigenartige Flora und Fauna näher kennenzulernen, und Geologen, denen die MacDonnell Ranges ein einzigartiges Betätigungsfeld boten. Ihnen folgten Prospektoren, die in der weiteren Umgebung von Stuart zahlreiche Bodenschätze fanden, vorab Gold-, Kupfer- und Glimmerlager, die heute zum Teil abgebaut werden. - In der vegetationsarmen Landschaft wurden Versuchsfarmen für Schaf- und Rinderzucht eingerichtet, und verschiedenerorts entstanden Missionsstationen und Wohnstätten von Aborigines. So entwickelte sich Stuart zu einem bedeutenden Mittelpunkt des südlichen Nordterritoriums mit zahlreichen zentralen Diensten. Es wurde zu einer Oase im Herzen Australiens.

\section{Beschwerliche Verkehrsverhältnisse}

Das größte Hindernis für eine rasche Entwicklung dieser Pioniersiedlung waren die beschwerlichen Zufahrtswege, denn es gab damals einzig die schlecht befahrbaren Straßen nach Oodnadatta im Süden, das seit 1891 Bahnendstation war, und nach der Hafenstadt Darwin an der Nordküste. Teilstrecken dieser Route wurden für den Transport der hölzernen Telegraphenstangen neu angelegt oder ausgebessert. Eine Reise von Oodnadatta nach Stuart dauerte mit dem Pferdewagen sechs, mit dem Reitpferd drei bis vier Tage. Immer mehr befaßten sich einflußreiche Persönlichkeiten mit dem Gedanken einer Süd-Nord-Bahnlinie. Aber weder Südaustralien, geschweige denn das von der Zentralregierung verwaltete Nordterritorium konnten sich zum Bau einer Bahn durch das praktisch unbesiedelte, wüstenähnliche Gebiet entschließen. Der finanzielle Aufwand schien in keinem annehmbaren Verhältnis zum zu erhoffenden Betriebserfolg zu stehen. Zwar bestanden 1891 bereits einige Bahnlinien, die als Teilstücke einer Süd-Nord-Verbindung in Frage kamen, so im Süden die $116 \mathrm{~km}$ lange Strecke Adelaide_Port Pirie mit Breitspur (1,60 m),

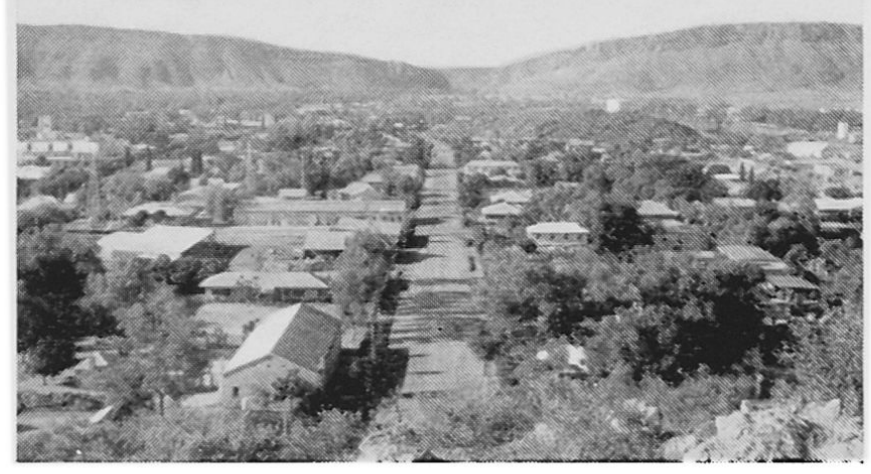

Alice Springs. Blick vom Anzac Hill gegen Süden. Im Vordergrund ein Teil des Shopping-Quartiers oder der «Kontaktzone». Rechts am Bildrand das Bahnhof- und Gewerbequartier. Bemerkenswert sind die vielen Bäume. die zum Teil nur dank künstlicher Bewässerung wachsen können. Im Hintergrund die MacDonnel Ranges mit der Klus Heavitree Gap, durch welche die Telegraphenleitung, die Straße und die Bahnlinie nach Süden führen. Die Aufnahme wurde am 20. Dezember 1968 morgens 7 Uhr gemacht. Zu dieser Tageszeit ruht der Straßenverkehr noch völlig Photo Werner Nigg

die $433 \mathrm{~km}$ lange Verbindung zwischen Port Pirie und Marree mit Normalspur $(1,43 \mathrm{~m})$ und die 398 $\mathrm{km}$ lange Fortsetzung nach Oodnadatta mit Schmalspur $(1,06 \mathrm{~m})$. Im Norden wurde 1917 das südlichste Stück der $508 \mathrm{~km}$ langen Schmalspurlinie $(1,06$ $\mathrm{m})$ von Darwin nach Birdum in Betrieb genommen. So fehlte noch die $1465 \mathrm{~km}$ lange Verbindung von Oodnadatta im Süden über Stuart nach Birdum.

Seit 1902 befaßte sich das Gouvernement des Commonwealth of Australia mit den Projekten der Central Australia Railway, denn nur der Bundesstaat konnte die Mittel für den Bau und die Anschaffungen aufbringen und die Garantie für die Deckung der wahrscheinlich zu erwartenden Betriebsdefizite übernehmen. Nach jahrelangen Verhandlungen mit Ingenieuren, Unternehmern, lokalen Verwaltungen und Interessengruppen wurde der Bau der Strecke Oodnadatta-Stuart in Angriff genommen, und 1929 konnte diese dem Betrieb übergeben werden.

\section{Aus Stuart wird Alice Springs}

Ein Jahr nach der Eröffnung der Bahnlinie wurde Stuart in Alice Springs umbenannt, zur Erinnerung an die erste Pionierzeit; vielleicht spielte bei der Wahl dieses Namens auch die Tatsache eine gewisse Rolle, daß Australien damals ein ausgesprochener "Männerstaat» war.

Bis heute blieb Alice Springs Endstation der Zentralaustralischen Eisenbahn, und deren Weiterfüh- 

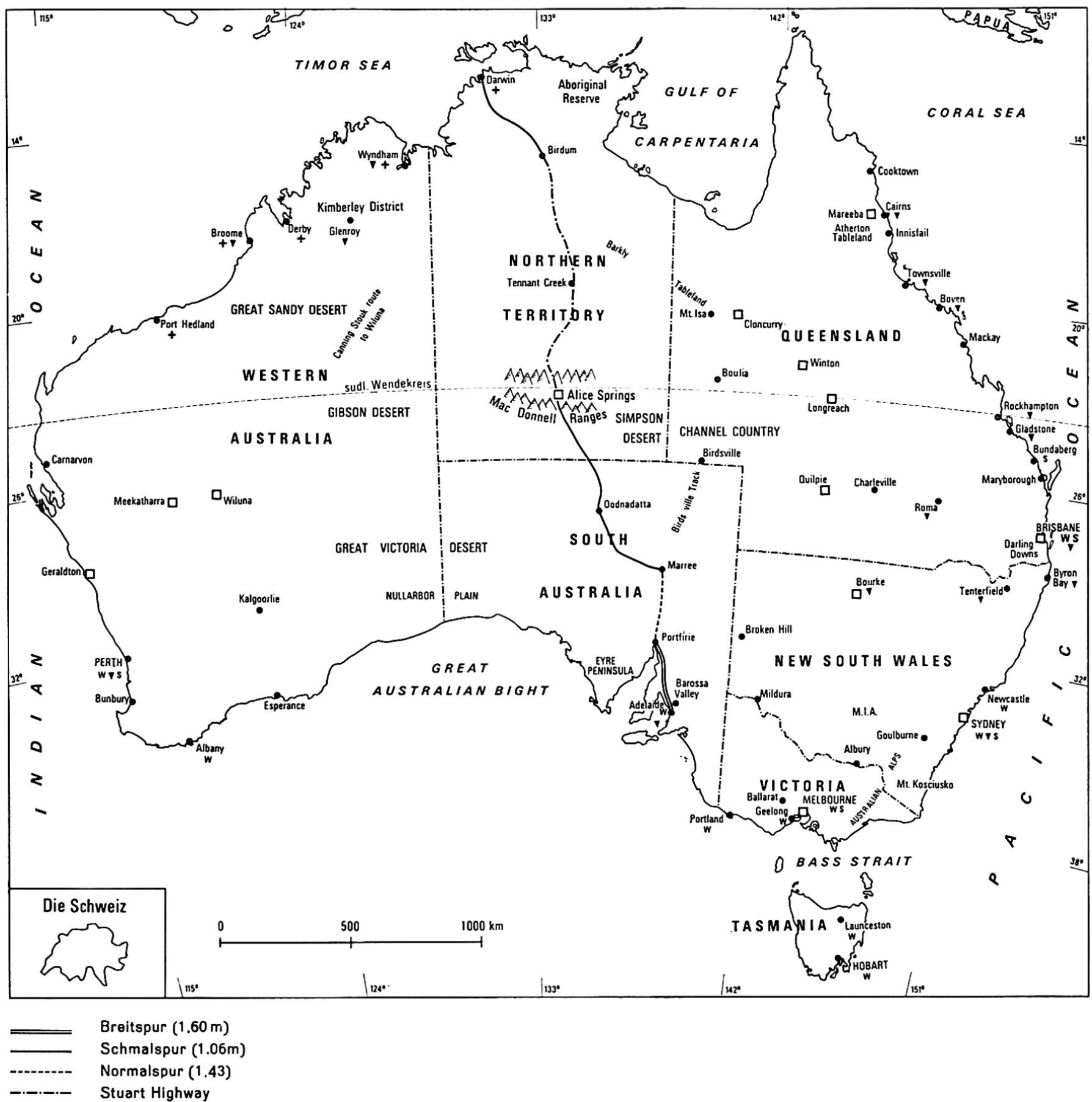

rung nach Birdum und damit die Fertigstellung der Süd-Nord-Linie wird wohl in absehbarer Zukunft nicht in Frage kommen; denn inzwischen ist das Zeitalter des Automobils und Flugzeugs angebrochen.

Heute führt eine gute Autostraße mit Hartbelag von Adelaide über Alice Springs nach Darwin, und der gesamte Güter- sowie ein Großteil des Personenverkehrs von Alice Springs nordwärts wickelt sich auf dem sogenannten Stuart Highway ab.

Neben zahlreichen Güterzügen, die teilweise noch von Dampflokomotiven gezogen werden (Australien besitzt billige eigene Kohle!), fahren zwischen Marree und Alice Springs in beiden Richtungen wöchentlich zwei von modernsten Dieselelektrolokomotiven gezogene Personenzüge mit gut eingerichteten, klimatisierten Schlaf-, Speise- und Auf- enthalts-( LLounge-»)Wagen. Eine Fahrt von Adelaide nach Alice Springs dauert (einschließlich des durch die verschiedenen Spurweiten bedingten zweimaligen Umsteigens) 31 Stunden. Trotz dieser langen Fahrzeit ist eine solche Bahnreise für jeden, der noch ein wenig Sinn für Abenteuer besitzt, ein selten schönes Erlebnis. Stundenlang zieht die lange Zugschlange gemächlich und schnurgerade durch steinige, rötliche Wüstenebenen. Auf langen Strekken bilden die Stangen der Telegraphenleitung die einzigen vertikalen Erscheinungen. Selten tauchen am fernen Horizont rundliche, graue Felsbuckel mit verschwommenen Umrissen auf. Einige der wenigen Bahnstationen sind nur an der Tafel mit dem Namen, einem zweiten Geleise und einer hölzernen Viehverladerampe erkennbar. Andere besitzen dazu noch ein kleines Stationsgebäude und zwei bis drei 
eingeschoßige Wohnhäuser, die von einem großen Wassertank überragt werden. Steigt man an diesen Stationen aus dem angenehm kühlen Zugswagen aus, dann schlägt einem eine wahre Backofenhitze ins Gesicht.

Eine Reise von Darwin nach Alice Springs mit Bahn und Autobus dauert 40 Stunden. Heute besitzt Alice Springs auch einen Flughafen für den zivilen Luftverkehr. Eine Flugreise von Adelaide oder Darwin nach Alice Springs dauert drei bis vier Stunden.

Als Endstation der Central Australia Railway ist Alice Springs der wichtigste Umschlagsplatz von Inneraustralien. Täglich werden große Mengen von Gütern, die von Süden mit der Bahn, von Norden mit Autolastzügen mit bis zu vier zweiachsigen Anhängern kommen, umgeladen.

Dank der modernen, raschen Verkehrsverbindungen wurde Alice Springs in den letzten Jahren immer mehr zum interessanten Touristenzentrum. Während des Südwinters, wenn es an den südlichen Badestränden zu kühl zum Baden ist, herrscht in Alice Springs angenehm warmes, sonniges Wetter. Neben der bereits erwähnten allgemeinen Großartigkeit der Landschaft mit ihren schönen, zum Teil bizarren Pflanzen und intensiven Farben gibt es in der näheren und weiteren Umgebung einmalige Naturwunder, so unter anderem die MacDonnell Ranges mit großartigen Felsformationen, Verwitterungsformen und Schluchten, wie zum Beispiel die Heavitree Gap, Fenn Gap, Tempe Bar Gap und Emily Gap; die riesigen, durch Verwitterung abgerundeten "Monolithen" Ayers Rock und The Olgas, die bei Morgen- und Abendbeleuchtung in zauberhaftem Rot erscheinen; der "Felszirkus» von Palm Valley und der Felsrücken Dancing Lubra Hill, die ihre Gesteinsschichten in seltener Schönheit präsentieren, sowie die enge Klamm Standley Chasm mit senkrecht aufsteigenden rotgelben Felswänden. Besondere Anziehungskraft üben auch die abgelegenen Camps oder Homesteads aus, wie zum Beispiel Ross River, die den Besuchern Pferde für unvergeßliche Ausritte zur Verfügung stellen. Ausflugsziele sind auch die Missionsstation Hermannsburg und verschiedene Eingeborenenreservate, unter anderem die Native Settlements Amoonguna, Haast Bluff und Yuendumu.

Aber auch in der Siedlung selbst oder in nächster Umgebung findet der Besucher viel Sehenswertes, so die alte Telegraphenstation, die in einem Nationalpark liegt, den Aussichtshügel Anzac Hill, der einen einzigartigen Uberblick über die Stadt bietet, und das Pitchi Richi Sanctuary mit eigenartigen naturalistischen Felsskulpturen eines Urbewohners. Alice Springs ist heute auch Verwaltungszentrum des Südteiles des Nordterritoriums. Es ist Sitz der einzigartigen Organisationen School of Air, einer Schule, die mit Hilfe des Radios und auf dem Korrespondenzweg die Kinder in den Hunderte von
Kilometern auseinanderliegenden Camps erfolgreich unterrichtet und der Flying Doctor Base, von wo aus der sehr gute und rasche ärztliche Notfalldienst für ganz Australien geleitet wird. Es wird behauptet, daß es Dank dieses Dienstes möglich sei, an jeden Punkt des Kontinentes innert 45 Minuten ärtzliche Hilfe zu senden. - Auf den Höhen der Mac-Donnell Ranges haben die amerikanischen Raumforschungsbehörden Stationen zur Beobachtung der künstlichen Satelliten errichtet.

Die Siedlung Alice Springs, die mit ganz wenig Ausnahmen aus eingeschoßigen Häusern besteht, ist schachbrettartig angeordnet und nimmt eine verhältnismäßig große Fläche ein - Bauland ist in Zentralaustralien noch keine "Mangelware»! Alice Springs läßt sich deutlich in einige Zonen gliedern. Östlich der Bahnstation liegt die "Kontaktzone» oder das Shopping Center mit Ladengeschäften, darunter verschiedenen Souvenirläden, Supermarkets, Warenhäusern, Hotels, Bars, Cafés, Reisebüros. Dort befinden sich auch verschiedene Kirchen, die Postbüros, verschiedene Verwaltungsgebäude und das Schwimmbad. - Südlich schließt sich das Viertel mit Schulen (darunter die Zentrale der School of Air), Spitälern, dem Hauptquartier der Flying Doctor Base und schönen Parkanlagen an. - Im Westen des Bahnhofs liegt die "Gewerbezone» mit Verladeeinrichtungen für Massen- und Stückgüter von Eisenbahnwagen auf Lastautos, mit Remisen, Garagen, Autoreparaturwerkstätten, Tankstellen, Park- und Lagerplätzen. Die Wohnviertel, deren schöne Häuser (alle ohne Keller) inmitten gepflegter Gärten stehen, liegen östlich des Todd Rivers, im Norden an der Ausfallstraße nach Darwin (Stuart Highway) und im Südwesten. Gegenwärtig (1969) wird für die Angestellten der amerikanischen Satellitenbeobachtungsstationen eine neue Wohnsiedlung gebaut.

Erwähnenswert ist vielleicht noch, daß die Straße, die zum östlichen Wohnquartier führt, quer durch das meist trockene Flußbett des Todd Rivers zieht Für die seltenen und kurzen Hochwasserzeiten besteht ein Fußgängersteg, dann ist der Automobilverkehr auf dieser Straße unterbrochen.

Heute zählt Alice Springs 7000 Einwohner; wenn die Entwicklung der letzten Jahre anhält - was zu erwarten ist - dann wird die Bevölkerungszahl in drei bis vier Jahren auf 10000 anwachsen. Neben der großen Mehrheit der anglo-australischen Bewohnen hat es verhältnismäßig viele Italiener, während die Zahl der Aborigines gering ist. Unter den Touristen sieht man neben vielen Australiern Ausländer aus allen Erdteilen.

So hat sich inmitten einer immensen, fast menschenleeren Landschaft innerhalb von sieben bis acht Dezennien aus einer weltabgeschiedenen Telegraphenstation die aufstrebende Siedlung Alice Springs entwickelt. 